\title{
Caveolin-1, encoded by CAV1, is differentially expressed in primary tumors and lymph
} node metastases in human breast cancer.

Shahan Mamoor, MS ${ }^{1}$

1shahanmamoor@gmail.com

East Islip, NY USA 11730

Breast cancer is the most frequently diagnosed cancer in women ${ }^{1}$. Metastasis is the major cause of death in patients diagnosed with cancer ${ }^{2}$. Prognosis for patients with breast cancer worsens as the number of axillary lymph nodes with metastasis increases ${ }^{3}$. We mined published microarray and mRNA quantitation datasets 4,5 to discover genes associated with metastasis to the lymph nodes, an early event in breast cancer metastasis. We found significant differential expression of the gene encoding caveolin-1, CAV1, when comparing primary breast tumors to lymph node metastases from women diagnosed with breast cancer. Analysis of a separate microarray dataset ${ }^{6}$ revealed that CAV1 was among the genes most differentially expressed when comparing primary tumors of the breast to adjacent normal breast tissue. Low expression of CAV1, in the upper but not lower survival quartile was associated with shorter recurrence-free and overall survival. CAV1 may be of relevance to processes underlying transformation or progression of the primary tumor in human breast cancer, as well as metastasis to the lymph nodes.

Keyword: breast cancer, breast cancer metastasis, lymph node metastasis, CAV1, caveolin-1, systems biology of breast cancer, targeted therapeutics in breast cancer. 
Invasive breast cancer is diagnosed in over quarter of a million women in the United States each year ${ }^{1}$. Metastasis is the most common cause of death in humans diagnosed with cancer $^{2}$. The lymphatic system is the primary route by which tumor cells traverse the body ${ }^{7}$. While patients with localized breast cancer are provided a 99\% 5-year survival rate, patients with regional breast cancer, cancer that has spread to lymph nodes or nearby structures, are provided an $86 \% 5$-year survival rate 8,9 . Patients with metastasis to distant sites are provided a $27 \% 5$ year survival rate 8,9 . We mined published microarray gene expression datasets 4,5 to discover genes associated with the primary tumor and lymph node metastasis in breast cancer and identified significant differential expression of caveolin-1, encoded by CAV1. CAV1 may be of relevance to breast cancer progression or metastasis to the lymph nodes.

\section{Methods}

We used datasets GSE1246484, GSE929775, and GSE1091696 for this differential gene expression analysis in conjunction with GEO2R. GSE124648 was generated using Affymetrix Human Genome U133A Array technology with $n=256$ primary breast tumors from patients with breast cancer and $n=67$ lymph node metastases from patients with breast cancer; platform GPL96 was used for this analysis. GSE92977, a multiplexed mRNA quantitation dataset, was generated using NanoString nCounter Nano105 Gene Expression Custom CodeSet technology with $n=123$ primary breast tumors from patients with breast cancer and $n=24$ lymph node metastases from patients with breast cancer; platform GPL17071 was utilized for this analysis. GSE109169 was generated using Affymetrix Human Exon 1.0 ST Array technology with $n=25$ adjacent control 
breast tissue and $n=25$ primary breast tumors from patients with breast cancer; platform GPL5175 was used for this analysis. The Benjamini and Hochberg method of $p$-value adjustment was used for ranking of differential expression but raw $p$-values were used to assess statistical significance of global differential expression. Log-transformation of data was autodetected, and the NCBI generated category of platform annotation was used. A statistical test was performed to evaluate whether CAV1 expression was significantly between primary breast tumors and lymph node metastases, or between adjacent control tissue and primary tumors of the breast using a two-tailed, unpaired t-test with Welch's correction. We used PRISM for all statistical analyses of differential gene expression in human breast cancer (Version 8.4.0)(455). For Kaplan-Meier survival analysis, we used the Kaplan-Meier plotter online tool $^{9}$ for correlation of CAV1 mRNA expression levels with overall survival in $n=3951$ and recurrencefree survival in $n=1402$ breast cancer patients, in total, and in upper and lower survival quartiles.

\section{Results}

We mined published microarray data $4,5,6$ to discover genes associated with progression of breast cancer: between normal breast tissue and primary tumors of the breast, and between primary breast tumors and metastasis of invasive breast cancer to the lymph nodes.

CAV1 is differentially expressed in lymph node metastases in invasive breast cancer.

We identified CAV1 as among the genes expressed most quantitatively differently, transcriptome-wide, when comparing primary tumors of the breast to lymph node metastases in

PAGE 3 OF 22 
women diagnosed with breast cancer ${ }^{4}$ (Table 1). When sorting each of the transcripts measured by microarray based on significance of change in expression between lymph node metastases and primary tumors of the breast, CAV1 ranked 165 out of 22283 total transcripts, equating to differential expression more significant than $99.3 \%$ of genes measured (Table 1). Differential expression of CAV1 in lymph node metastases in human breast cancer was statistically significant (Table $1 ; p=5.09 \mathrm{E}-43$ ).

Analysis of a second mRNA multiplexed quantitation dataset ${ }^{5}$ again revealed differential expression of CAV1 in lymph node metastases when compared to primary breast tumors (Table 2). When sorting each of the transcripts measured by microarray based on significance of change in expression between primary tumors of the breast lymph node metastases, CAV1 ranked 24 out of 105 transcripts measured, equating to differential expression more significant than $77.1 \%$ of genes measured in this code set (Table 2). Differential expression of CAV1 in lymph node metastases in human breast cancer in this second dataset was statistically significant (Table 2; $p=3.59 \mathrm{E}-02)$.

CAV1 is differentially expressed in primary tumors of the breast in invasive breast cancer.

Analysis of a third microarray dataset ${ }^{6}$ revealed that CAV1 was also among the genes most differentially expressed in primary tumors of the breast from women diagnosed with invasive breast cancer when compared to adjacent normal breast tissue (Table 3). When sorting each of the transcripts measured by microarray based on significance of change in expression between primary tumors of the breast and adjacent, benign breast tissue, CAV1 ranked 33 out 
of 19076 transcripts measured, equating to differential expression more significant than $99.8 \%$ of genes measured (Table 3). Differential expression of CAV1 in primary tumors of the breast in human breast cancer was statistically significant (Table $3 ; p=7.85 \mathrm{E}-18$ ).

CAV1 is expressed at significantly lower levels in lymph node metastases when compared to primary tumors of the breast.

We obtained exact mRNA expression levels of the differentially expressed CAV1 transcript to directly compare CAV1 expression between primary tumors of the breast and lymph node metastases. We also performed a statistical test to evaluate significance of difference in CAV1 expression between primary tumors of the breast and lymph node metastases was statistically significant. CAV1 was expressed at lower levels in lymph node metastases as compared to primary tumors of the breast; this difference in CAV1 mRNA expression was statistically significant (Figure $1 ; p<0.0001$ ). We calculated a fold change of $0.8485 \pm 0.1872$ and $0.9024 \pm 0.1697$ in CAV1 mRNA expression in lymph node metastases relative to primary breast tumors (Table 1 and Table 2, respectively).

CAV1 is expressed at significantly lower levels in primary tumors of the breast when compared to adjacent benign breast tissue.

We also obtained exact mRNA expression levels of the differentially expressed CAV1 transcript to directly compare CAV1 expression between primary tumors of the breast and adjacent, benign breast tissue. We also performed a statistical test to evaluate significance of difference in CAV1 expression between breast tumors and benign breast tissue in women with invasive breast cancer. CAV1 was expressed at lower levels in primary breast tumors; this 
difference in mRNA expression of CAV1 was statistically significant (Figure $3 ; p<0.0001$ ). We calculated a fold change of $0.8 \pm 0.1$ in CAV1 mRNA expression in primary tumors of the breast relative to adjacent benign breast tissue (Table 3).

CAV1 expression in primary breast tumors is significantly correlated with median overall survival.

We performed Kaplan-Meier survival analysis ${ }^{9}$ using CAV1 mRNA expression in primary breast tumors coupled with paired overall survival data from each patient to determine whether CAV1 tumor expression was correlated with survival outcomes in breast cancer. Patients whose tumors expressed lower levels of CAV1 possessed shorter recurrence-free and overall survival than patients with high tumor expression of CAV1 (Figure 4). Median overall survival (OS) of patients in the low expression cohort was 81.6 months, while median OS in patients in the high CAV1 expression cohort was 120 months (Table 4); Median recurrence-free survival (RFS) of patients in the low expression cohort was 216.66 months, while median RFS in patients in the high CAV1 expression cohort was 228.85 months (Table 5); difference in median OS based on CAV1 tumor expression in breast cancer was statistically significant (Figure 4; logrank $p$-value: 0.047 ; hazard ratio: $0.81(0.65-1))$ as was difference in RFS in breast cancer based on CAV1 tumor expression (Figure 5; logrank p-value: 0.12; hazard ratio: 0.87 $(0.78-0.97))$. It is important to note, however, that for both RFS and OS Kaplan-Meier analysis in all patients, the false discovery rate was unacceptably high: $20 \%$ for RFS and greater than $50 \%$ for OS. This could be attributable to a number of different factors.

PAGE 6 OF 22 
Similarly, median overall survival (OS) of patients in the low expression cohort was 86 months in patients in the upper survival quartile, while median OS in patients in the high CAV1 expression cohort was 169.2 months in the upper survival quartile (Table 4); Median recurrence-free survival (RFS) of patients in the low expression cohort was 44.4 months in the upper survival quartile, while median RFS in patients in the high CAV1 expression cohort was 62.88 months in the upper survival quartile (Table 5); difference in median OS based on CAV1 tumor expression in breast cancer in patients in the upper quartile was statistically significant (Figure 4; logrank $p$-value: 0.031 ; hazard ratio: $0.75(0.57-0.98)$ ) as was difference in RFS in breast cancer in patients in the upper quartile based on CAV1 tumor expression (Figure 5; logrank $p$-value: 0.00051 ; hazard ratio: $0.79(0.69-0.9))$

Thus, we found that caveolin-1 was among the genes whose expression was most significantly different in lymph node metastases when compared to primary tumors of the breast, and in primary tumors of the breast when compared to normal breast tissue. Caveolin-1 expression correlated with overall and recurrence-free survival; patients with low caveolin-1 expressed possessed shorter survival than those with high caveolin-1 expression.

\section{Discussion}

Mice deficient in caveolin-1 are absent of caveolae, invaginated areas of the plasma membrane, demonstrating requirement for caveolin-1 in generation of caveolae ${ }^{11}$. Downstream consequences of caveolin-1 deficiency and lack of caveolae was impairment of calcium and nitric oxide signaling, resulting in defects of contraction and relaxation of heart muscle, and 
perseveration of endothelial cell proliferation resulting in alveolar septum thickening in the lungs ${ }^{11}$. A study identifying high-affinity interaction partners of fatty acids in 3T3-L1 adipocytes found caveolin-1 was a fatty acid binding protein ${ }^{12}$. In endothelial cells growing in culture (HMEC-1 cells), exposure to angiogenic growth factors including vascular endothelial growth factor (VEGF), platelet-derived growth factor (PDGF) and hepatocyte growth factor (HGF) resulted in decreased expression of caveolin-1 in sub-confluent but not confluent cells ${ }^{13}$. Caveolin-1 was important for endothelial tubule formation and its expression could be observed occurring prior to formation of endothelial tubules on Matrigel ${ }^{13}$. Consistent with a role for caveolin-1 in generation of endothelial tubes, ectopic expression of caveolin-1 resulted in increased formation of tubules in HMEC-1 cells ${ }^{13}$. A separate study found that the oncogene vAbl could induce expression of caveolin-1, indicating that oncogenic stimuli could support caveolin expression ${ }^{14}$. This appeared to be a cellular defense response against transformation as opposed to supporting transformation, as expression of caveolin-1 in NIH3T3 cells impaired cellular transformation in soft agar and induced apoptosis in F11 cells ${ }^{14}$. Caveolin-1 can also function as a sort of an adaptor, linking together $\beta 1$ integrins to the Fyn kinase; integrin ligation by Fyn results in recruitment of Shc and activation of the extracellular signal-related kinase (ERK) important for cell cycle progression, suggesting that caveolin-1 is required for induction anchorage-dependent cell growth through integrin signaling ${ }^{15}$. Thus, among its many roles, caveolin-1 functions as an integral component of plasma membrane microdomain structures known as caveolae, is a fatty-acid binding protein, can influence angiogenesis and endothelial tubule formation, and is described as critical for anchorage-dependent cell growth. 
Multiple studies have previously implicated caveolin-1 in the biology of breast cancer, including other solid tumors such as sarcoma. While expressed at relatively high levels in fibromatoses, leiomyomas, hemangiomas and lipomas compared to mesenchymal tissues, caveolin-1 expression was decreased in 4 of 6 liposarcomas, 17 of 20 leiomyosarcomas, 16 of 16 myxoid/round cell/pleomorphic liposarcomas, 5 of 8 angiosarcomas, 15 of 18 malignant fibrous histiocytomas, and 8 of 8 synovial sarcomas. Importantly, ectopic expression of caveolin-1 in HT-1080 fibrosarcoma cells resulted in marked reduction in colony formation in vitro ${ }^{16}$. One study found mutation of the CAV1 gene in $16 \%$ of human breast cancers surveyed ${ }^{17}$. The resulting mutation, P132L, has oncogenic properties as its expression can transform NIH 3T3 cells in vitro ${ }^{17}$. P132L functions in a dominant-negative manner, preventing proper sub-cellular localization of wild-type caveolin-1 to caveolae ${ }^{18}$. Critically, specific genetic deletion of CAV1 in mice resulted in intraductal hyperplasia in the mammary gland, suggesting that caveolin- 1 can function to prevent mammary epithelial cell hyperplasia in vivo ${ }^{18}$. Caveolin-1 is expressed at lower levels in highly metastatic 4T1.2 and 4T1.13 tumors as compared to non-metastatic tumors, which is consistent with our analysis of published microarray data from human breast cancers ${ }^{19}$. Similarly, high expression of caveolin-1 resulted in decreased metastasis to the lungs and to the bones when expressed in $4 \mathrm{~T} 1.2$ cells grafted in mice ${ }^{19}$. Loss of caveolin-1 expression in the tumor stroma was associated with disease recurrence, tumor stage, and consistent with our analysis of published microarray data, metastasis to the lymph nodes ${ }^{20}$. In breast cancer patients treated with tamoxifen, loss of caveolin-1 expression in the storm predicted worse patient outcome suggestive of tamoxifen resistance 20. 
Of critical importance, and again consistent with our analysis of microarray data from primary tumors, loss of caveolin-1 expression in the stroma predicted 11.5-fold reduction in the 5-year progression-free survival rate for breast cancer patients considered "lymph-node positive" or with metastasis to the lymph nodes ${ }^{20}$. Clearly, multiple studies support our analysis of published microarray data based on analysis of multiple microarray and multiplexed gene expression datasets $4,5,6$ that reduced or lost expression of caveolin- 1 is associated with metastasis to the lymph nodes in human breast cancer and can predict or is correlated with worse patient outcomes.

By mining independently published gene expression data $4,5,6$, we found that the gene encoding caveolin-1, CAV1, was among the genes most differentially expressed in human breast cancer, both in lymph node metastases when compared to primary tumors of the breast as well as in primary tumors of the breast as compared to adjacent benign breast tissue. CAV1 appears to be expressed in a gradient-like fashion in invasive breast cancer, and may be of relevance to processes underlying progression of breast cancer from transformation to spread of breast cancer to the lymph nodes.

PAGE 10 OF 22 


\section{References}

1. DeSantis, C.E., Ma, J., Gaudet, M.M., Newman, L.A., Miller, K.D., Goding Sauer, A., Jemal, A. and Siegel, R.L., 2019. Breast cancer statistics, 2019. CA: a cancer journal for clinicians, 69(6), pp.438-451.

2. Mehlen, P. and Puisieux, A., 2006. Metastasis: a question of life or death. Nature reviews cancer, 6(6), pp.449-458.

3. Fisher, B., Bauer, M., Wickerham, D.L., Redmond, C.K., Fisher, E.R., Cruz, A.B., Foster, R., Gardner, B., Lerner, H., Margolese, R. and Poisson, R., 1983. Relation of number of positive axillary nodes to the prognosis of patients with primary breast cancer. An NSABP update. Cancer, 52(9), pp.1551-1557.

4. Sinn, B.V., Fu, C., Lau, R., Litton, J., Tsai, T.H., Murthy, R., Tam, A., Andreopoulou, E., Gong, Y., Murthy, R. and Gould, R., 2019. SET ER/PR: a robust 18-gene predictor for sensitivity to endocrine therapy for metastatic breast cancer. NPJ breast cancer, 5(1), pp.1-8.

5. Cejalvo, J.M., de Dueñas, E.M., Galván, P., García-Recio, S., Gasión, O.B., Paré, L., Antolin, S., Martinello, R., Blancas, I., Adamo, B. and Guerrero-Zotano, Á., 2017. Intrinsic subtypes and gene expression profiles in primary and metastatic breast cancer. Cancer research, 77(9), pp.2213-2221.

6. Chang, J.W., Kuo, W.H., Lin, C.M., Chen, W.L., Chan, S.H., Chiu, M.F., Chang, I.S., Jiang, S.S., Tsai, F.Y., Chen, C.H. and Huang, P.H., 2018. Wild-type p53 upregulates an early onset breast cancer-associated gene GAS7 to suppress metastasis via GAS7-CYFIP1-mediated signaling pathway. Oncogene, 37(30), pp.4137-4150.

7. Rahman, M. and Mohammed, S., 2015. Breast cancer metastasis and the lymphatic system. Oncology letters, 10(3), pp.1233-1239.

8. ACS Cancer Facts \& Figures 2019. https://www.cancer.net/cancer-types/breast-cancermetastatic/statistics.

9. Survival Rates for Breast Cancer. https://www.cancer.org/cancer/breast-cancer/ understanding-a-breast-cancer-diagnosis/breast-cancer-survival-rates.html

10. Györffy, B., Lanczky, A., Eklund, A.C., Denkert, C., Budczies, J., Li, Q. and Szallasi, Z., 2010. An online survival analysis tool to rapidly assess the effect of 22,277 genes on breast cancer prognosis using microarray data of 1,809 patients. Breast cancer research and treatment, 123(3), pp.725-731.

PAGE 11 OF 22 
11. Drab, M., Verkade, P., Elger, M., Kasper, M., Lohn, M., Lauterbach, B., Menne, J., Lindschau, C., Mende, F., Luft, F.C. and Schedl, A., 2001. Loss of caveolae, vascular dysfunction, and pulmonary defects in caveolin-1 gene-disrupted mice. science, 293(5539), pp.2449-2452.

12. Trigatti, B.L., Anderson, R.G. and Gerber, G.E., 1999. Identification of caveolin-1 as a fatty acid binding protein. Biochemical and biophysical research communications, 255(1), pp. 34-39.

13. Liu, J., Wang, X.B., Park, D.S. and Lisanti, M.P., 2002. Caveolin-1 expression enhances endothelial capillary tubule formation. Journal of Biological Chemistry, 277(12), pp. 10661-10668.

14. Engelman, J.A., Wykoff, C.C., Yasuhara, S., Song, K.S., Okamoto, T. and Lisanti, M.P., 1997. Recombinant expression of caveolin-1 in oncogenically transformed cells abrogates anchorage-independent growth. Journal of Biological Chemistry, 272(26), pp.16374-16381.

15. Wary, K.K., Mariotti, A., Zurzolo, C. and Giancotti, F.G., 1998. A requirement for caveolin-1 and associated kinase Fyn in integrin signaling and anchorage-dependent cell growth. Cell, 94(5), pp.625-634.

16. Wiechen, K., Sers, C., Agoulnik, A., Arlt, K., Dietel, M., Schlag, P.M. and Schneider, U., 2001. Down-regulation of caveolin-1, a candidate tumor suppressor gene, in sarcomas. The American journal of pathology, 158(3), pp.833-839.

17. Hayashi, K., Matsuda, S., Machida, K., Yamamoto, T., Fukuda, Y., Nimura, Y., Hayakawa, T. and Hamaguchi, M., 2001. Invasion activating caveolin-1 mutation in human scirrhous breast cancers. Cancer research, 61(6), pp.2361-2364.

18. Lee, H., Park, D.S., Razani, B., Russell, R.G., Pestell, R.G. and Lisanti, M.P., 2002. Caveolin-1 mutations (P132L and null) and the pathogenesis of breast cancer: caveolin-1 (P132L) behaves in a dominant-negative manner and caveolin-1 (-/-) null mice show mammary epithelial cell hyperplasia. The American journal of pathology, 161(4), pp. 1357-1369.

19. Sloan, E.K., Stanley, K.L. and Anderson, R.L., 2004. Caveolin-1 inhibits breast cancer growth and metastasis. Oncogene, 23(47), pp.7893-7897.

20. Witkiewicz, A.K., Dasgupta, A., Sotgia, F., Mercier, I., Pestell, R.G., Sabel, M., Kleer, C.G., Brody, J.R. and Lisanti, M.P., 2009. An absence of stromal caveolin-1 expression predicts early tumor recurrence and poor clinical outcome in human breast cancers. The American journal of pathology, 174(6), pp.2023-2034.

PAGE 12 OF 22 


\section{Table 1: Differential expression of CAV1 in lymph node metastases in breast cancer.}

Rank of differential expression, $\mathrm{p}$-value of global differential expression, $\mathrm{t}$, a moderated $\mathrm{t}$ statistic, B, the log-odds of differential expression between the groups compared, fold change of CAV1 in lymph node metastases as compared to primary breast tumors, and gene are listed in this chart. 
PAGE 14 OF 22 


\begin{tabular}{|c|c|c|c|c|c|c|c|}
\hline Rank & ID & $p$-value & $\mathbf{t}$ & B & FC & Gene & Gene name \\
\hline 33 & 3020302 & $7.85 \mathrm{E}-18$ & $-1.3 \mathrm{E}+01$ & 30.348134 & $0.8 \pm 0.1$ & CAV1 & caveolin 1 \\
\hline
\end{tabular}

Table 3: Differential expression of CAV1 in primary tumors of the breast in breast cancer.

Rank of differential expression, $\mathrm{p}$-value of global differential expression, $\mathrm{t}$, a moderated $\mathrm{t}$ statistic, B, the log-odds of differential expression between the groups compared, fold change of CAV1 in primary tumors of the breast as compared to adjacent benign breast tissue, and gene are listed in this chart. 
Figure 1: CAV1 is expressed at significantly lower levels in lymph node metastases as compared to primary tumors of the breast.

The mRNA expression level of CAV1 in primary tumors of the breast from women diagnosed with invasive breast cancer (left) and in lymph node metastases from women diagnosed with breast cancer (right) is graphically depicted with the result of a statistical test evaluating significance of difference in CAV1 expression between primary tumors and lymph node metastases, a $p$-value, listed above.

PAGE 16 OF 22 


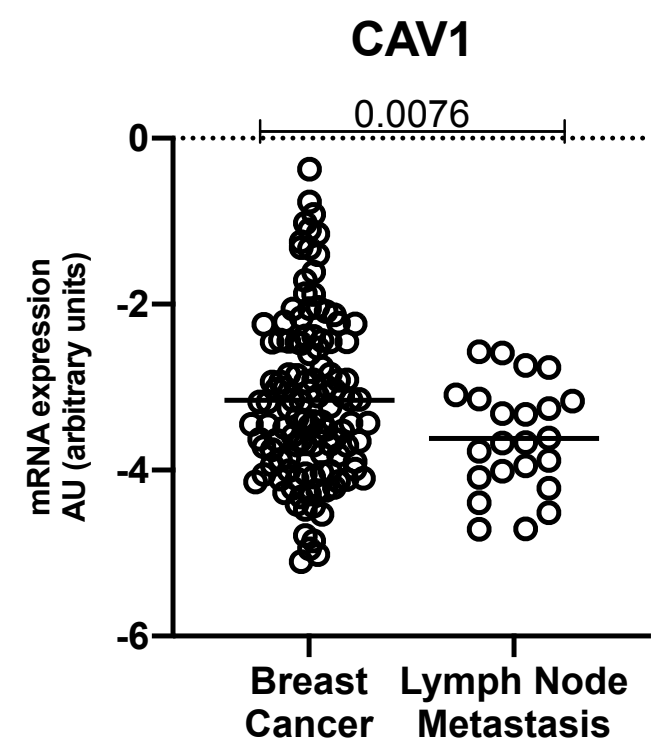

Figure 2: CAV1 is expressed at significantly lower levels in lymph node metastases as compared to primary tumors of the breast.

The mRNA expression level of CAV1 in primary tumors of the breast from women diagnosed with invasive breast cancer (left) and in lymph node metastases from women diagnosed with breast cancer (right) is graphically depicted with the result of a statistical test evaluating significance of difference in CAV1 expression between primary tumors and lymph node metastases, a $p$-value, listed above. 


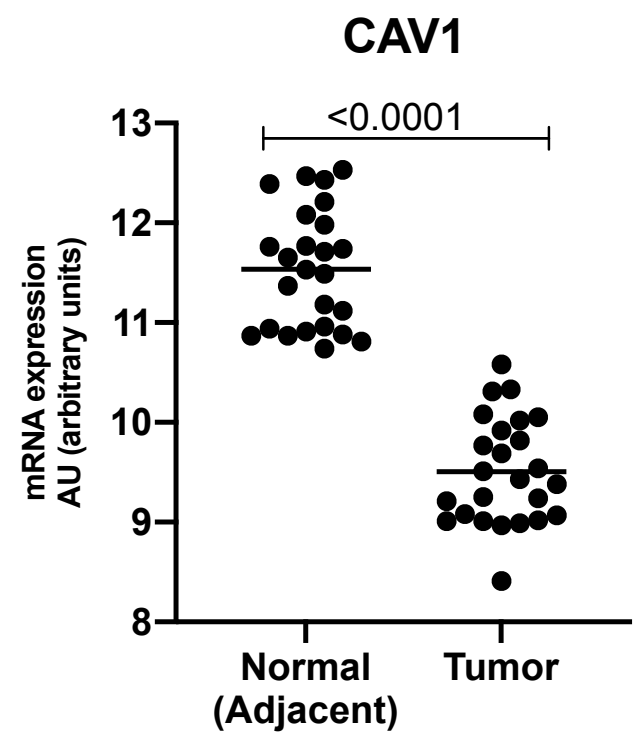

Figure 3: CAV1 is expressed at significantly lower levels in primary tumors of the breast as compared to benign adjacent breast tissue.

The mRNA expression level of CAV1 in benign, tumor-adjacent breast tissue (left) and in primary tumors of the breast (right) is graphically depicted with the result of a statistical test evaluating significance of difference in CAV1 expression between normal breast tissue and primary tumors of the breast, a $p$-value, listed above.

PAGE 18 OF 22 
A

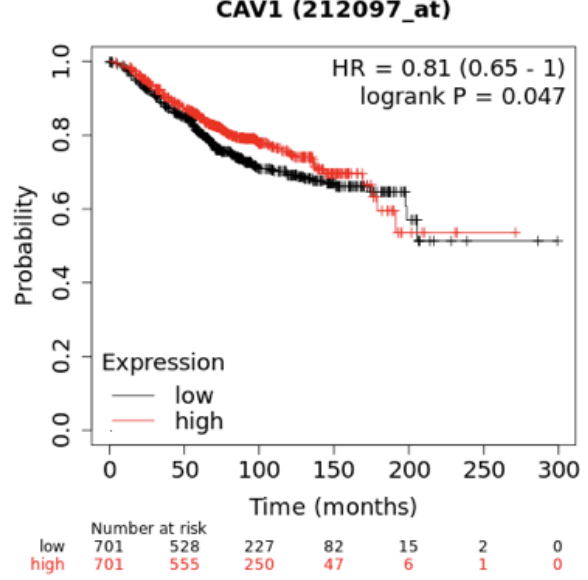

B

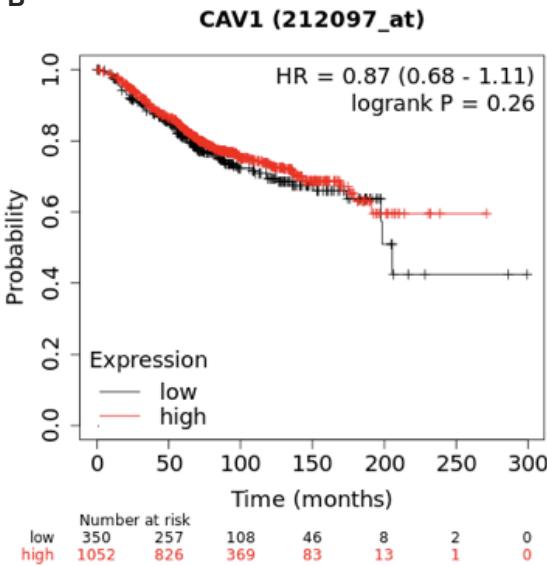

C
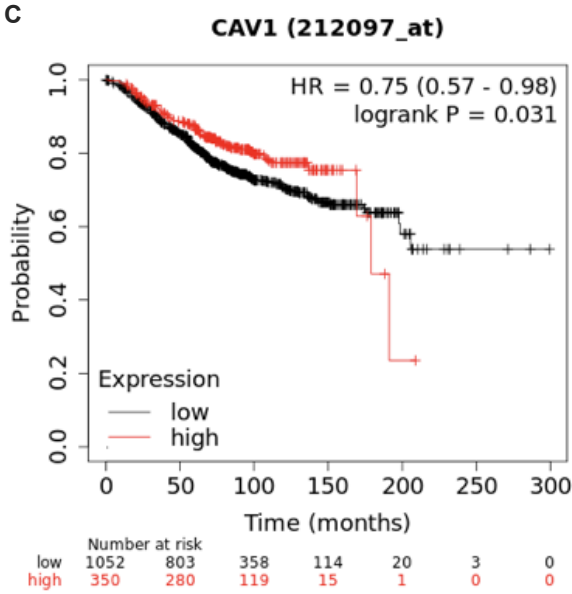

Figure 4: CAV1 expression in breast tumors is significantly correlated with overall survival in patients with breast cancer, as a whole and in patients in the upper survival quartile, but with a high false-discovery rate.

Depicted in this Kaplan-Meier plot is the probability of overall survival for $n=3951$ total patients stratified into two groups, based on low or high expression of CAV1 in patient primary tumors. Analysis is performed for all patients (A), patients in the lower survival quartile (B), and patients in the upper survival quartile $(\mathrm{C})$. The log rank $p$-value denoting statistical significance of difference in overall survival when comparing the two groups, as well as hazard ratio for this comparison is listed above. Listed below is the number of patients at risk (number of patients alive) per interval, after stratification based on CAV1 expression; in the first interval, number at risk is number of patients alive; in each subsequent interval, number at risk is the number at risk less those who have expired or are censored. The false-discovery rate for RFS in all patients was greater than $50 \%$.

PAGE 19 OF 22 
2

3

4

5

6

7

8

9

10

11

12

13

14

15

16

17

18

19

20

21

22

23

24

25

26

27

28

Table 4: CAV1 expression in breast tumors significantly correlates with patient survival.

The median overall survival of $n=1402$ breast cancer patients based on stratification into low or high tumor expression of LHFP is listed in this chart, in two patient populations: encompassing all patients, or encompassing only the upper survival quartile.

High expression cohort (months)

\begin{tabular}{|c|c|c|}
\hline & $\begin{array}{c}\text { Low expression } \\
\text { cohort (months) }\end{array}$ & $\begin{array}{c}\text { High expression } \\
\text { cohort (months) }\end{array}$ \\
\hline All patients & 81.6 & 120 \\
\hline Upper quartile & 86 & 169.2 \\
\hline
\end{tabular}


A CAV1 (212097_at)

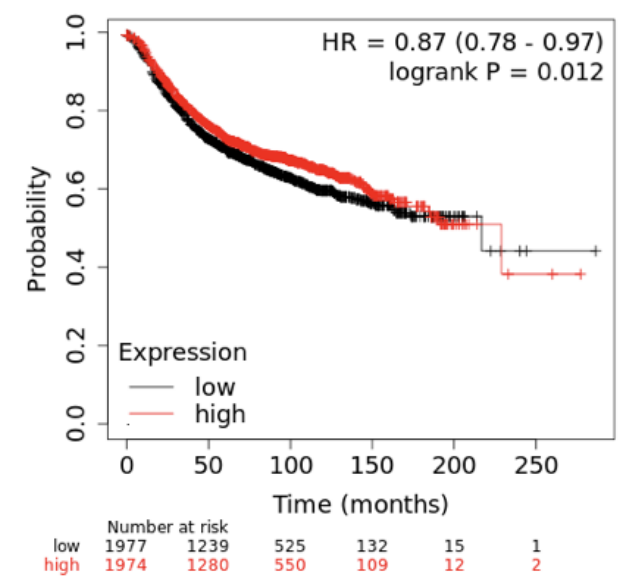

B

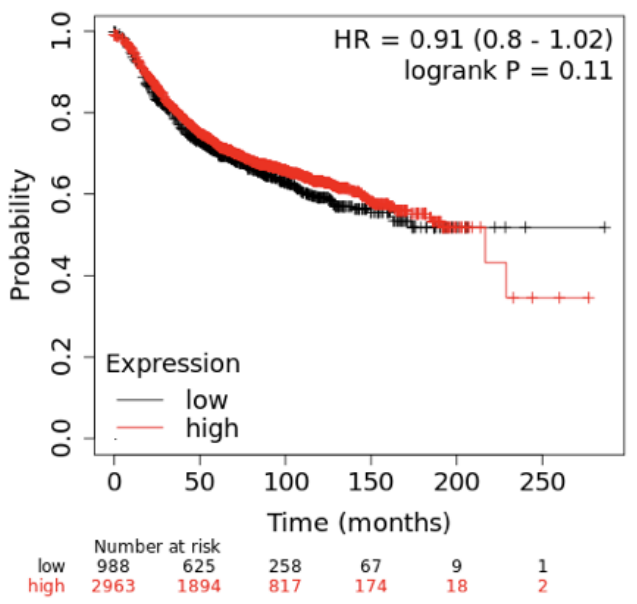

C CAV1 (212097_at)

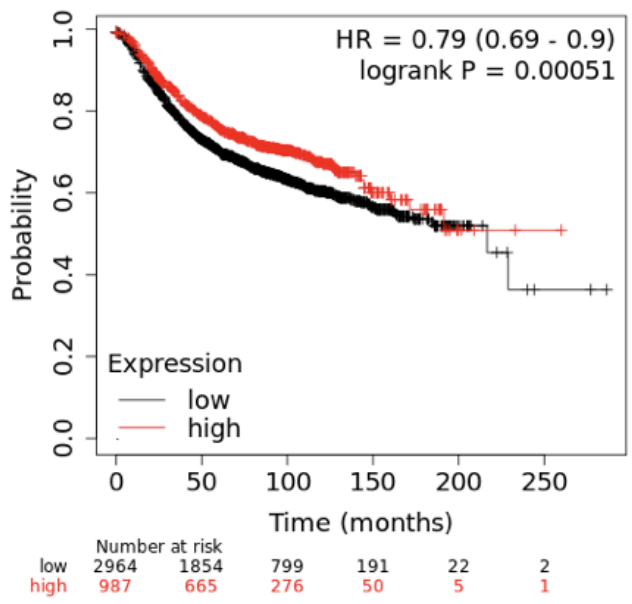

Figure 5: CAV1 expression in breast tumors is significantly correlated with recurrence-free survival in patients with breast cancer, as a whole and in patients in the upper survival quartile, but with a high false-discovery rate.

Depicted in this Kaplan-Meier plot is the probability of recurrence-free survival (RFS) for $n=3951$ total patients stratified into two groups, based on low or high expression of CAV1 in patient primary tumors. Analysis is performed for all patients (A), patients in the lower survival quartile (B), and patients in the upper survival quartile (C). The log rank $p$-value denoting statistical significance of difference in overall survival when comparing the two groups, as well as hazard ratio for this comparison is listed above. Listed below is the number of patients at risk (number of patients alive) per interval, after stratification based on CAV1 expression; in the first interval, number at risk is number of patients alive; in each subsequent interval, number at risk is the number at risk less those who have expired or are censored. The false-discovery rate for RFS in all patients is $20 \%$. 


\begin{tabular}{|c|c|c|}
\hline & $\begin{array}{c}\text { Low expression } \\
\text { cohort (months) }\end{array}$ & $\begin{array}{c}\text { High expression } \\
\text { cohort (months) }\end{array}$ \\
\hline All patients & 216.66 & 228.85 \\
\hline Upper quartile & 44.4 & 62.88 \\
\hline
\end{tabular}

Table 5: CAV1 expression in breast tumors significantly correlates with patient survival.

The median overall survival of $n=1402$ breast cancer patients based on stratification into low or high tumor expression of LHFP is listed in this chart, in two patient populations: encompassing all patients, or encompassing only the upper survival quartile. 